Results Significant improvement in the postoperative quality of life in the majority of such patients, with lowered perioperative morbidity and added advantage of better cosmesis was seen. The extent of unwanted and avoidable drilling of the mastoid cortex could be significantly lowered. Functional results in terms of postoperative hearing was found to be improved with closure of $A B$ gap to the extent of $30 \mathrm{~dB}$ in majority of patients.

Conclusion Despite the technical demand of therapeutic intervention for delicate middle ear sound conducting mechanism by the transcanal endoscopic approach, it has been seen to stand the test of time as a technique of minimal invasive surgical approach. The functional preservation of the middle ear mucosa and reestablishment of the ventilation pathways are the keys to successful outcome in the management of mucosal disease of the middle ear cleft.

Clinical Significance The clinical significance of the original research study is to ascertain the essence of endoscopic minimal access surgical techniques for the management of isolated mucosal diseases of the middle ear cleft and its long-term results.

\section{A0006: Over Expression of Psoriasin in Middle Ear Cholesteatoma Increases Inflammation, Bone Destruction, and Severity of Disease: Ultrastructural and Molecular Findings}

A. K. Kairo, ${ }^{1}$ J.A. Quadri, ${ }^{2}$ A. Singh,, ${ }^{1}$ S. Sarwar, ${ }^{2}$ R. Kumar, ${ }^{1}$

A. Thakar, ${ }^{1}$ A. Shariff, ${ }^{2}$ S.C. Sharma ${ }^{1}$

${ }^{1}$ Department of Otorhinolaryngology and Head and Neck Surgery, All India Institute of Medical Sciences, New Delhi, India ${ }^{2}$ Department of Anatomy, All India Institute of Medical Sciences, New Delhi, India

Introduction Cholesteatoma remains a mystery as its trigger is still not known. How insidiously tympanic membrane starts to accumulate keratin and start to invade surrounding structure is still unknown. No treatment of this disease is available to stop progression except surgery.

Aim The study was performed to evaluate the role of psoriasin and proinflammatory cytokines in progression of middle ear cholesteatoma.

Methodology Study was conducted in AIIMS, New Delhi, after ethical approval. Eighteen (12 cases of cholesteatoma tissue as study group and six cases of PSRP as control taken after tympanomastoidectomy) patients were included in the study after taking written consent. Clinical aggressiveness was evaluated on table under operative microscope by evaluation of erosion of ossicles, invasion of surrounding structures, complications, if any, associated in preoperative period. Cholesteatoma cases were divided into two groups based on severity of the disease (moderate and severe). The cases of PSRP (... please add one line about and extended form of it ...), describe a condition in which a part of the eardrum lies deeper within the ear than its normal position, were taken as control. The middle ear cholesteatoma tissues were processed for histopathological, ultrastructural, and immunohistochemical analyses.
Results Hematoxylin and eosin-stained tissue sections showed stratified squamous epithelium, keratinous contents, ghost squamous cells, and inflammatory cells. The psoriasin expression in cholesteatoma tissues was significantly higher as compared with posterior superior retraction pocket (PSRP). A Psoriasin expression increases with the severity of the disease. The proinflammatory cytokines (IL-6 and IL-1 $\beta$ ) were also upregulated along with VEGF and MMP-9 in cholesteatoma cases compared with control and the expression increased with disease severity. TEM images of ultra-thin sections of the tissues showed strips of stratified squamous epithelium layer along with inflammatory and apoptotic cells. A very unique ultrastructural feature of bacterial localization within the membrane pocket was seen, which gave direct evidence of bacterial infection in the middle ear cholesteatoma.

Acknowledgment Authors are thankful to the Electron Microscope Facility for providing an opportunity to conduct ultrastructural analysis, and AIIMS, New Delhi for providing financial (Intramural Research Funding) and all other supports.

\section{A0007: Repair of Focal Posterior Canal Wall Defects Using Bony Plate over the Sigmoid Sinus \\ Devika Shere}

Aim To study the effectiveness of repair of focal posterior canal wall defects using bony plate over the sigmoid sinus.

Materials and Methods We performed a retrospective review of seven cases operated at our tertiary care center between January 2015 and August 2017 for repair of posterior canal wall defects.

All seven patients had focal defects in posterior canal wall. These defects were repaired by using bony plate harvested from sigmoid plate after an intact canal wall mastoidectomy. The repair was done after a through clearance of active pathology in the ear in all of these cases. These cases were followed up for a period of 6 months to 1 year.

Results All seven cases had successful repair of the posterior canal wall defect. There was good epithelialization of the external auditory canal and the reconstruction was stable. All of these patients had relief from intermittent otorrhea.

Conclusion This technique of repair is simple, repeatable, and provides an effective physiological reconstruction of the posterior canal wall. Bony sigmoid plate is invariably easily available. The plate of bone has a natural curvature of the canal wall. And in terms of tissue quality, the sigmoid bony plate and posterior canal wall plate were similar.

Clinical Significance In an intact canal wall (canal wall up procedure) inadvertent defect of canal wall must be repaired to avoid intermittent otorrhea and to prevent retraction pockets formation. This technique of repair fulfills this requirement and is cost-effective with good patient compliance. 